\title{
Ability of Brain Natriuretic Peptide Tests and Homocysteine to Exclude Congestive Heart Failure
}

\author{
Mira Barak ${ }^{1}$, Jorge E. Schliamser ${ }^{2}$, Nisan Yaniv $^{3}$ and Paul Froom*,1 \\ ${ }^{I}$ Regional Laboratory of Haifa and Western Galilee, Clalit Health Services, Nesher, Israel; ${ }^{2}$ Department of Cardiovas- \\ cular Medicine, Lady Davis Carmel Medical Center, Haifa, Israel; ${ }^{3}$ Department of Cardiology, Lin Medical Center, \\ Clalit Health Services, Haifa, Israel
}

\begin{abstract}
Among the most promising tests for early detection of congestive heart failure (CHF) are brain natriuretic peptides (BNPs), but it is unclear if homocysteine can aid in differentiating those with and without disease. We attempt to use a combination of tests to reduce the need for echocardiography without missing any cases of CHF.

We compared $50 \mathrm{CHF}$ patients with an ejection fraction of less than $60 \%$, to 100 patients with non-specific symptoms but with a normal echocardiogram. After setting sensitivity at $100 \%$, specificities for BNP, amino-terminal probrain natriuretic peptide (NT-pro BNP), and homocysteine were $34 \%, 28 \%$ and $36 \%$ respectively. Combinations of two tests if homocysteine was included increased the specificity to $56 \%$. The AUC for BNP and NT-proBNP were not significantly different (92.8 and 95.9, respectively) whereas the AUC for BNP and NT-proBNP tests were superior to homocysteine (83.2). Compared with performing echocardiography in all patients, savings were dependent on the pretest probability. At a $1 \%$ pretest probability of CHF, savings were $44.5 \%$ while using a combination of NT-proBNP with homocysteine test. Homocysteine can be used in combination with either of the BNPs to decrease costs of early detection of CHF.
\end{abstract}

Keywords: Homocysteine, brain natriuretic peptide, NT-proBNP, congestive heart failure, costs, comparison.

\section{INTRODUCTION}

Congestive heart failure (CHF) is a major health problem with inherent morbidity and increased mortality rates. If early detection and treatment of asymptomatic patients with CHF decrease the rate of progression to symptomatic disease [1], tests to rule out disease could be appropriate [2]. Echocardiography is the standard method for detecting CHF, but resources might not allow its widespread use since it is expensive and its performance and interpretation require expertise [3]. Furthermore there may be cost savings if relatively inexpensive blood tests were available with the ability to reliably rule out CHF.

Among the variety of tests, the most promising blood tests are the brain natriuretic peptides: brain natriuretic peptide (BNP) [1,4-9], and amino-terminal probrain natriuretic peptide (NT- proBNP) [8,10,11]. Plasma homocysteine might also contribute to ruling out $\mathrm{CHF}$, since homocysteine has been shown to be a risk factor for developing CHF, and plasma levels are associated with the severity of the disease $[12,13]$. We are unaware however of studies comparing the ability of homocysteine with that of brain natriuretic peptides to identify patients with CHF, without missing any cases of disease.

In the following study we assess and compare the ability of these three blood tests alone and in combination to reduce

*Address correspondence to this author at the Clalit Health Services, Regional Laboratory of Haifa and the Western Galilee, 47 Hata'asia St., Nesher, 20300, Israel; Tel: 972-4-6394470; Fax: 972-4-6243302;

E-mail: paulfr@clalit.org.il the need for echocardiography without missing any cases of CHF.

\section{METHODS}

\section{Patients}

The study was approved by the Ethics Committee of Lady Davis Carmel Medical Center, and Informed consent was obtained from each patient. We compared two groups of patients referred for echocardiography. The study group included 50 patients with a history of treatment for CHF and an EF of less than $60 \%$ at the time of testing. Forty of the 50 patients had an EF at time of blood testing of less than $40 \%$, where the others were between $45-55 \%$. They were compared to 100 consecutive patients with nonspecific symptoms, such as shortness of breath either at rest or on exercise, chronic dry cough, leg swelling, or severe fatigue but with an $\mathrm{EF}$ of $60 \%$ or more [14], and no history of treatment for CHF.

\section{Materials}

Blood samples were drawn by vacutainer EDTA plastic tubes (Greiner bio-one, Kremsmuenster, Austria), and centrifuged within 4 hours. Plasma samples were aspirated and transferred into plastic tubes that were stored at $-70^{\circ} \mathrm{C}$ until analysis.

BNP was assayed by Abbott BNP immunoassay that was preformed on Axsym analyser according to manufacturer's recommendations; Abbott (Wiebaden-Delekenheim, Germany). NT-proBNP was performed on Immulite 2000 ac- 
cording to manufacturers instructions; DPC (Los Angele, CA).

Homocysteine was detected by Abbott Homocysteine Fluorescence Polarization Immunoassay that was preformed on Axsym analyser according to manufacturer's recommendations; Abbott (Wiebaden-Delekenheim, Germany).

\section{Statistical Analysis}

We used MedCalc (Version 11.1.1, MedCalc Software, Mariakerke, Belgium) for statistical analysis. Mean values of the various tests in the two groups were compared with a one sided t-test, a more sensitive method to detect significant differences in the expected direction. A p value of less than 0.05 was considered significant. Next, the cut off value for each test was determined by fixing the sensitivity at $100 \%$. Given $100 \%$ sensitivity, we considered combinations of parallel tests to determine if specificity might be increased. Then we used the given specificities to calculate the possible cost savings that might be obtained without missing any patients with congestive heart failure. We assumed that an echocardiography costs 420 US\$ [15], BNP test 32 US\$ [15], NT pro-BNP test 20 US\$ [9], and homocysteine test 22 US\$ [16] and calculated costs at different pretest probabilities and savings comparing the use of blood tests with selective use of echocardiography to the use of echocardiography on all patients. We also compared the areas under the receiver operator curve (AUC) for the three tests in order to compare the overall ability of the tests to differentiate between those with and without CHF at all possible cut-off values.

\section{RESULTS}

Age was similar in the two groups, whereas for all the tests, mean values were significantly higher in the patients with CHF (Table 1). After setting the cut-off value to give a $100 \%$ sensitivity, the specificities for BNP, NT-pro BNP, and homocysteine were $34 \%, 28 \%$ and $36 \%$ respectivity (Table 2). Combinations of two tests if homocysteine was included increased the specificity to $56 \%$, and with three tests $63 \%$. The AUC for BNP and NT-proBNP were not significantly different $(92.8,95 \%$ confidence interval (CI) $=87.4-96.4$, and $95.9,95 \%$ CI 91.3-98.4 respectively) whereas the AUC for BNP and NT-proBNP tests were superior to homocysteine, $83.2 \%$ (95\% CI 76.2-88.8, $\mathrm{p}=0.026$ and $\mathrm{p}=0.002$ respectively). If we used a cutoff of $\mathrm{EF}<45 \%$ to define CHF, then the specificity of the NT-proBNP assay was increased from $28 \%$ to $84 \%$, and the BNP assay from $34 \%$ to $83 \%$, whereas the specificity for homocysteine was not improved.

Savings were dependent on the pretest probability, with a savings of $44.5 \%$ using a combination of NT-proBNP and homocysteine tests at $1 \%$ pretest probability which decreased to $18 \%$ if the pretest probability was $50 \%$ (Table 3 ). If the cost of echocardiography decreased to around $120 \$$, there would be no savings (calculation not shown).

\section{DISCUSSION}

We found that the specificity of homocysteine is comparable to the brain natriuretic peptides in identifying those without $\mathrm{CHF}$ after requiring $100 \%$ sensitivity. Our results are consistent with previous studies showing that homocysteine is a risk factor for CHF as well as being associated with

Table 1. Comparison of the Various Tests in those with and without Congestive Heart Failure

\begin{tabular}{|c|c|c|c|c|}
\hline Variables & Units & No CHF N=100 & CHF N=50 & P value \\
\hline \hline Age & years & $67.9 \pm 1.1^{*}$ & $68.7 \pm 1.6$ & 0.6913 \\
\hline Homocysteine & $\mu \mathrm{mol} / \mathrm{L}$ & $11.5 \pm 0.5$ & $18.5 \pm 0.7$ & $<0.001$ \\
\hline BNP & $\mathrm{ng} / \mathrm{L}$ & $86 \pm 48$ & $744 \pm 68$ & $<0.001$ \\
\hline NT pro BNP & $\mathrm{ng} / \mathrm{L}$ & $155 \pm 261$ & $3424.5 \pm 370$ & $<0.001$ \\
\hline
\end{tabular}

*Mean \pm standard error.

Table 2. Comparing the Specificities in the Best Differentiating Tests Using a Cutoff of $100 \%$ Sensitivity

\begin{tabular}{|c|c|c|c|}
\hline Test & Specificity (\%) N=100 & Specificity (\%) 95\% CI & Cut-off Value \\
\hline \hline BNP & 34 & $24.8-44.2$ & $24.8 \mathrm{ng} / \mathrm{L}$ \\
\hline NT-pro BNP & 28 & $19.5-37.9$ & $34.4 \mathrm{ng} / \mathrm{L}$ \\
\hline Homocysteine & 36 & $26.6-46.2$ & $9.61 \mu \mathrm{mol} / \mathrm{L}$ \\
\hline NT-proBNP+ BNP* & 44 & $34.1-54.3$ & \\
\hline BNP+ homocysteine & 56 & $45.7-65.9$ & \\
\hline NT-proBNP + homocysteine & 56 & $45.7-65.9$ & $52.8-72.4$ \\
\hline BNP+NT-proBNP+ homocysteine & 63 & & \\
\hline
\end{tabular}

*Compared to other two combinations individually $(\mathrm{p}=0.08)$ 
Table 3. Cost Analysis for Diagnosing and Ruling Out Congestive Heart Failure with Various Combinations of Blood Tests and Echocardiograms (US Dollars)

\begin{tabular}{|c|c|c|c|c|c|c|}
\hline Pretest & Tests & Costs Times Number & $\begin{array}{c}\text { Echocardiogram } \\
\text { Total Number }\end{array}$ & $\begin{array}{c}\text { Costs of } \\
\text { Echo }^{\dagger}\end{array}$ & $\begin{array}{l}\text { Total } \\
\text { Costs }\end{array}$ & Savings $\%$ \\
\hline \multirow[t]{2}{*}{$1 \%$} & $1 *$ & $22 \times 200=4400$ & $127+2=129$ & 54180 & 58580 & $31.3 \%$ \\
\hline & 2 & $42 \times 200=8400$ & $87+2=89$ & 37380 & 45780 & $44.5 \%$ \\
\hline \multirow[t]{3}{*}{$5 \%$} & 1 & $22 \times 200=4400$ & $122+10=132$ & 55440 & 59840 & $28.8 \%$ \\
\hline & 2 & $42 \times 200=8400$ & $84+10=94$ & 39480 & 47880 & $43.1 \%$ \\
\hline & 3 & $74 \times 200=14800$ & $70+10=80$ & 33600 & 48400 & $42.4 \%$ \\
\hline \multirow[t]{3}{*}{$50 \%$} & 1 & $22 \times 200=4400$ & $63+100=163$ & 68460 & 72860 & $13.3 \%$ \\
\hline & 2 & $42 \times 200=8400$ & $44+100=144$ & 60480 & 68880 & $18.0 \%$ \\
\hline & 3 & $74 \times 200=14800$ & $37+100=137$ & 57540 & 72340 & $13.9 \%$ \\
\hline
\end{tabular}

*Assuming specificities of $36 \%$ for one test (homocysteine), $56 \%$ for two tests (one of the brain natriuretic peptides and homocysteine), and $63 \%$ for three tests.

${ }^{\dagger}$ Cost of blood tests in order of addition is $22 \$$ for homocysteine, $20 \$$ for NT- proBNP, and 32\$ for BNP $=$ together $74 \$$. cost of echocardiogram $=\$ 420$.

Number of echocardiograms is calculated by assuming that all patients with disease are positive by screening and therefore the number $=$ the prevalence, whereas the number of tests for those without disease is dependent on the one minus the specificity (e.g. $200 \mathrm{X} \mathrm{1 \%}=2$, and 1- 0.36 times 198 (those without disease) $=127$ ); thus the total number of echocardiographs here $=127+2$ or 129 .

the severity of the disease $[12,13,17]$. However it is not unexpected that the AUC for homocysteine although high $(83 \%)$, was significantly less than that of the Brain natriuretic peptides, since levels are not specific and have been shown to correlate with serum folate, B12, and creatinine levels [18]. The cutoff level of $9.6 \mu$ mole/L for homocysteine is slightly lower than that suggested as the lower relative value for those with good vitamin status $(12 \mu \mathrm{mole} / \mathrm{L})$ [12].

Our study is not directly comparable to most other studies that used EF cutoff values of $30-50 \%$ [4] to define CHF. We used a cutoff of less than $60 \%$ in order to insure that patients with mild congestive heart failure were not included in the control group. This would be likely to decrease specificity of the tests. In fact if we used a cutoff of $\mathrm{EF}<45 \%$ to define CHF, then the specificity of our NT-proBNP assay increased from $28 \%$ to $84 \%$, the BNP from $34 \%$ to $83 \%$. At $100 \%$ sensitivity other studies found variable BNP specificities of around $30 \%$ [7], close to zero [6], 40\%[8] and $19.6 \%[15]$, whereas for NT-proBNP a specificity of around $70 \%$ was found in one study [8] and $61 \%$ in another [19]. The variable results are probably due to patient selection and might also be due to differences in the various BNP assays [20]. Nevertheless our cutoff value for BNP of $24.8 \mathrm{ng} / \mathrm{L}$ is similar to that of other studies using different BNP assays and definitions of normal cardiac function [7,19], but would have increased to $71 \mathrm{ng} / \mathrm{L}$ if CHF was defined as an EF of less than $45 \%$. For NT-proBNP a cutoff value of $358 \mathrm{ng} / \mathrm{L}$ was reported in one study [19] whereas we found a very low value of 34.4 that increased to 185 if abnormal cardiac func- tion was defined as less than $45 \%$. Further studies with large numbers of patients are clearly needed to more precisely define appropriate cutoff values.

We also found that a combination of NT-proBNP with homocysteine can lead to $44.5 \%$ savings compared to doing echocardiography in all patients with a pretest prevalence of $1 \%$, but savings decrease with increased pretest prevalence. There would be no savings if echocardiography with a cost of $120 \$$ per test was widely available.

Our results should be interpreted with caution. Methodological differences might lead to different results. Furthermore our selection of patients with CHF might represent a spectrum bias with $80 \%$ having an EF of less than $40 \%$. Specificity might be lower if more patients with EFs of between $40-60 \%$ are included. Still our results may be relevant to clinical practice since an EF of less than $40 \%$ is often used to determine when it is appropriate to treat asymptomatic patients in order to prevent progression to symptomatic $\mathrm{CHF}$ [1]. Finally the Israeli population eats a Mediterranean diet rich in vitamins and extrapolation to other populations with different diets might not be warranted with regards to homocysteine.

There is no consensus on recommending screening of asymptomatic populations or case finding in those with nonspecific symptoms. Randomized controlled trials are needed to determine if there is decreased morbidity and/or mortality in those found with CHF treated earlier than in the control group. Our study suggests that plasma homocysteine should 
be included in the screening/case finding tests for such a study.

\section{ACKNOWLEDGEMENTS}

We thank Abbott (Wiebaden-Delekenheim, Germany) that provided the kits for BNP and homocysteine. We also thank DPC (Los Angele, CA) for providing kits for NTproBNP.

\section{REFERENCES}

[1] Heidenreich, P.A.; Gubens, M.A.; Fonarow, G.C.; Konstam, M.A.; Stevenson, L.W.; Shekelle, P.G. Cost effectiveness of screening with B-type Natriuretic peptide to identify patients with reduced left ventricular ejection fraction. J. Am. Coll. Cardiol., 2004, 43, 1019-1026.

[2] Consensus, P. BNP Consensus Panel 2004: A clinical approach for the diagnostic prognostic, screening, treatment monitoring and therapeutic roles of natriuretic peptides in cardiovascular diseases. Congestive Heart Fail., 2004, 10, 1-27.

[3] Appleton, C.P.; Firstenberg, M.S.; Garcia, M.J.; Thomas, J.D. The echo-Doppler evaluation of left ventricular diastolic function: a current perspective. Cardiol. Clin., 2000, 18, 513-546.

[4] Battaglia, M.; Pewsner, D.; Juni, P.; Egger, M.; Bucher, H.C.; Bachmann, L.M. Accuracy of B-type natriuretic peptide tests to exclude congestive heart failure: systematic review of test accuracy studies. Arch. Intern. Med., 2006, 166, 1073-1080.

[5] Lukowicz, T.V.; Fischer, M.; Hense, H.W.; Doring, A.; Stritzke, J.; Riegger, G.; Schunkert, H.; Luchner, A.; MONICA Investigators. BNP as a marker of diastolic dysfunction in the general population: Importance of left ventricular hypertrophy. Eur. J. Heart Fail., 2005, 7, 525-531.

[6] Yamamoto, K.; Burnett, J.C.; Bermudez, E.A.; Jougasaki, M.; Bailey, K.R.; Redfield, M.M. Clinical criteria and biochemical markers for the detection of systolic dysfunction. J. Card. Fail., 2000, 6,194-200.

[7] Aspromonte, N.; Ceci, V.; Chiera, A.; Coletta, C.; D'Eri, A.; Feola, M.; Giovinazzo, P.; Milani, L.; Noventa, F.; Scardovi, A.B.; Sestili, A.; Valle, R. Rapid brain natriuretic peptide test and Doppler echocardiography for early diagnosis of mild heart failure. Clin. Chem., 2006, 52, 1802-1808.

[8] Costello-Boerrigter, L.C.; Boerrigter, G.; Redfield, M.M.; Rodeheffer, R.J.; Urban, L.H.; Mahoney, D.W.; Jacobsen, S.J.; Heublein, D.M.; Burnett, J.C. Jr. Amino-terminal pro-B-type natriuretic peptide and B-type natriuretic peptide in the general community: de- terminants and detection of left ventricular dysfunction. J. Am. Coll. Cardiol., 2006, 47, 345-353.

[9] Siebert, U.; Januzzi, J.L.; Beinfeld, M.T.; Cameron, R.; Gazelle, GS. Cost-effectiveness of using N-Terminal Pro-BNP to guide the diagnostic assessment and management of dyspneic patients in the Emergency Department. Am. J. Cardiol., 2006, 98, 800-805.

[10] Yeo, K.T.; Dumont, K.E.; Brough, T. Elecsys NT-ProBNP and BNP assays: are there analytically and clinically relevant differences? J. Card. Fail., 2005, 11(5 Suppl), S84-8.

[11] Jortani, S.A.; Prabhu, S.D.; Valdes, Jr, R. Strategies for developing biomarkers of heart failure. Clin. Chem. 2004, 50, 265-278.

[12] Herrmann, M.; Taban-Shomal, O.; Hubner, U.; Bohm, M.; Herrmann, W. A review of homocysteine and heart failure. Eur. J. Heart Fail., 2006, 8, 571-576.

[13] Herrmann, M.; Kindermann, I.; Muller, S.; George, T.; Klindermann, M.; Bohm, M.; Herrmann, W. Relationship of plasma homocysteine with the severity of chronic heart failure. Clin. Chem., 2005, 51, 1512-1515.

[14] Hayashi, Y.; Takeuchi, M.; Takaoka, H.; Hata, K.; Mori, M.; Yokoyama, M. Alteration in energetics in patients with left ventricular dysfunction after myocardial infarction: increased oxygen cost of contractility. Circulation, 1996, 93, 932-939.

[15] Ng, L.L.; Pathik, B.; Loke, I.W.; Squire, I.B.; Davies, J.E. Myeloperoxidase and C-reactive protein augment the specificity of B-type natriuretic peptide in community screening for systolic heart failure. Am. Heart J., 2006, 152, 94-101.

[16] Nallamothu, B.K.; Fendrick, A.M.; Rubenfire, M.; Saint, S.; Bandekar, R.R.; Omenn, G.S. Potential clinical and economic effects of homocyst(e)ine lowering. Arch. Intern. Med., 2000, 160, 3406-3412

[17] Vasan, R.S.; Beiser, A. D'Agostino, R.B.; Levy, D.; Selhub, J.; Jacques, P.F.; Rosenberg, I.H.; Wilson, P.W. Plasma homocysteine and risk for congestive heart failure in adults without prior myocardial infarction. JAMA, 2003, 289, 1251-1257.

[18] Raeder, S.; Landaas, S.; Laake, K.; Lyberg, T.; Engedal, K. Homocysteine measurements in geriatric patients. Scand. J. Clin. Lab. Invest., 2006, 66, 309-315.

[19] Vanderheyden, M.; Bartunek. M.; Claeys, G.; Manoharan, G.; Beckers, J.F.; Ide, L. Head to head comparison of N-terminal proB-type natriuretic peptide and B-type natriuretic peptide in patients with/without left ventricular systolic dysfunction. Clin. Biochem., 2006, 39, 640-645.

[20] Barak, M.; Weinberger, R.; Marcusohn, J.; Froom, P. Harmonization of the Bayer ADVIA Centaur and Abbott AxSYM automated B-type natriuretic peptide assay in patients on hemodialysis. Clin. Chem. Lab. Med., 2005, 43, 554-556. 